\title{
Trends in Hyperglycemic Crisis Hospitalizations and in- and out-of-Hospital Mortality in the Last Decade Based on Korean National Health Insurance Claims Data
}

\author{
Ji Hong You ${ }^{1}$, Sun Ok Song ${ }^{1}$, Se Hee Park ${ }^{1}$, Kyoung Hye Park ${ }^{1}$, Joo Young Nam ${ }^{1}$, Dong Wook Kim², Hyun Min Kim³, \\ Dong-Jun Kim ${ }^{4}$, Yong-ho Lee ${ }^{5}$, Byung-Wan Lee ${ }^{5}$ \\ ${ }^{1}$ Division of Endocrinology and Metabolism, Department of Internal Medicine, ${ }^{2}$ Department of Policy Research Affairs, \\ National Health Insurance Service Ilsan Hospital, Goyang; ${ }^{3}$ Division of Endocrinology and Metabolism, Department of Internal \\ Medicine, Chung-Ang University College of Medicine, Seoul; ${ }^{4}$ Department of Internal Medicine, Inje University Ilsan Paik \\ Hospital, Inje University College of Medicine, Goyang; ${ }^{5}$ Division of Endocrinology and Metabolism, Department of Internal \\ Medicine, Severance Hospital, Yonsei University College of Medicine, Seoul, Korea
}

Background: Hyperglycemic crisis is a metabolic emergency associated with diabetes mellitus. However, accurate epidemiologic information on cases of hyperglycemic crisis in Korea remains scarce. We evaluated trends in hyperglycemic crisis hospitalizations and in- and out-of-hospital mortality in Korea. We also predicted future trends.

Methods: We extracted claims data with hyperglycemic crisis as the principal diagnosis from the National Health Insurance Service database in Korea from January 2004 to December 2013. We investigated the numbers of claims with hyperglycemic crisis and identified trends in hyperglycemic crisis based on those claims data. We predicted future trends by statistical estimation.

Results: The total annual number of claims of hyperglycemic crisis increased from 2,674 in 2004 to 5,540 in 2013. Statistical analysis revealed an increasing trend in hyperglycemic crisis hospitalizations $(P$ for trend $<0.01$ ). In contrast, the hospitalization rate per 1,000 diabetes cases showed a decreasing trend $(P$ for trend $<0.01)$ during this period. The mortality rate per 1,000 diabetes cases also showed a decreasing trend $(P$ for trend $<0.0001$ ). However, no distinct linear trend in the case-related fatality rate at $<60$ days over the last decade was observed. The predicted number of annual claims of hyperglycemic crisis will increase by 2030 .

Conclusion: The number of hyperglycemic crisis hospitalizations in Korea increased in the last decade, although the hospitalization rate per 1,000 diabetes cases and mortality rate decreased. Also, the predicted number of annual claims will increase in the future. Thus, it is necessary to establish long-term healthcare policies to prevent hyperglycemic crisis.

Keywords: Hyperglycemic crisis; Epidemiology; Diabetic ketoacidosis; Hyperglycemic hyperosmolar nonketotic coma

\section{INTRODUCTION}

Hyperglycemic crisis is a serious metabolic emergency associated with uncontrolled diabetes mellitus (DM). It can be subcat-

Received: 28 May 2019, Revised: 1 July 2019, Accepted: 3 July 2019

Corresponding author: Sun Ok Song

Division of Endocrinology and Metabolism, Department of Internal Medicine, National Health Insurance Service Ilsan Hospital, 100 Ilsan-ro, Ilsandong-gu, Goyang 10444, Korea

Tel: +82-31-900-3470, Fax: +82-31-900-0519, E-mail: songso@nhimc.or.kr egorized diabetic ketoacidosis (DKA), hyperosmolar hyperglycemic state (HHS), and a mixed state of acidosis and hyperosmolarity [1]. DKA is an extreme metabolic state caused by insulin deficiency, in which ketone bodies are produced by the

Copyright $\odot 2019$ Korean Endocrine Society

This is an Open Access article distributed under the terms of the Creative Commons Attribution Non-Commercial License (http://creativecommons.org/ licenses/by-nc/4.0/) which permits unrestricted non-commercial use, distribution, and reproduction in any medium, provided the original work is properly cited. 
breakdown of fatty acids. HHS, previously referred to as hyperosmolar hyperglycemic nonketotic coma, is defined as the presence of high blood sugar in patients with high serum osmolarity without significant ketoacidosis.

Hyperglycemic crisis results in significant morbidity or death in patients with DM. DKA is the most common cause of death in children and adolescents with type 1 DM [2]. The in-hospital mortality rate of DKA in the United States is $<1 \%$ overall in patients with DM [3]. Higher mortality rates have been reported in elderly patients and in those with life-threatening illnesses [4]. A retrospective cohort study in Scotland reported DKA mortality rates after intensive care unit admission of $8 \%, 18 \%$, and $35 \%$ at 30 days, 1 year, and 5 years, respectively [5]. The HHS mortality rate is significantly higher than that of DKA, with recently reported rates of $10 \%$ to $20 \%$ [6].

Hyperglycemic crisis is a major challenge in the healthcare system because it has a high cost of treatment and high fatality rate, imposing an enormous cost burden and requiring massive resources. In the United States, DKA is responsible for more than 500,000 hospital days per year, with estimated annual direct and indirect medical expenses of approximately 2.4 billion United States dollar in 2004 [7,8]. Hospitalizations for DKA steadily increased in the United States from 2009 to 2014 among all age groups, at an average annual rate of $6.3 \%$ [3].

Because of the cost burden and high fatality rate of hyperglycemic crisis, it is important to investigate its actual epidemiologic status and associated medical costs. However, accurate information on hyperglycemic crisis in Korea is currently limited. To obtain a more accurate understanding of its epidemiologic status, it is essential to use reliable population-based data, such as healthcare claims data. Healthcare claims data, which are widely used in epidemiology and health services research, constitute a large population-based longitudinal registry [9]. These data can be used to directly and indirectly determine the current status of hyperglycemic crisis, and on the basis of such findings, it is possible to predict future trends.

Therefore, using nationwide healthcare claims data over the last decade, we aimed to evaluate trends in hyperglycemic crisis hospitalizations and in- and out-of-hospital mortality in Korea, both to characterize the current epidemiologic state of hyperglycemic crisis and to predict future trends.

\section{METHODS}

\section{Data source}

A compulsory National Health Insurance Service (NHIS) pro- gram was launched by the Korean government in February 1999. As of 2013, the NHIS program covered the entire population of Korea (51 million individuals). The database analyzed in this study was derived from health insurance claims in the NHIS claims database, from which the NHIS provided data after deidentification. The claims case data contained patients' date of birth, sex, region of residence, and date of hospitalization. The study protocol was reviewed and approved by the National Health Insurance Service Ilsan Hospital Institutional Review Board (approval number: NHIMC 2015-02-031). Written informed consent by the patients was waived due to a retrospective nature of our study.

\section{Study population}

Using NHIS claims data from January 1, 2004, to December 31, 2013, we extracted information on patients with DM and hyperglycemic crisis for each year. Patients with DM were defined as those with at least one claim with a principal or first additional diagnosis of DM [10]. The principal and first additional diagnoses were determined according to the International Classification of Diseases, Tenth Revision, Clinical Modification (ICD10-CM) codes for diabetes (E1000-E1090, type 1 diabetes; E1100-E1190, type 2 diabetes; E1200-E1290, malnutrition-related diabetes; E1300-E1390, diabetes associated with other disease, other diabetes; and E1400-E1490, diabetes type unknown, unknown diabetes). We also included patients with hyperglycemic crisis as the principal or first additional diagnosis at the time of discharge among patients who were admitted to the emergency room. Patients with hyperglycemic crisis were defined as those with claims in which the principal or first additional diagnosis was DM with coma (E1X0X) or DM with acidosis (E1X1X). For example, E1000 is the code for type $1 \mathrm{DM}$ with coma and hyperosmolarity, while E1010 is the code for type $1 \mathrm{DM}$ with ketoacidosis. The data were extracted on August 30, 2015. For more detailed subanalyses, the patients were stratified by sex and age group ( 0 to 9,10 to 19,20 to 29,30 to 39,40 to 49,50 to 59,60 to 69,70 to 79 , and $>80$ years).

\section{Statistical analysis}

All data were analyzed using SAS software version 9.3 (SAS Institute Inc., Cary, NC, USA). The annual hyperglycemic crisis hospitalization rates from 2004 to 2013 were calculated by dividing the number of patients with hyperglycemic crisis by the total number of subjects with DM. The annual mortality rates due to hyperglycemic crisis were calculated by dividing the total number of deaths after hospitalization for hyperglycemic cri- 
sis by the total number of patients with DM. The annual caserelated fatality rates at $<60$ days were calculated by dividing the number of case-related fatalities at $<60$ days by the total number of patients with DM. The annual trends in the number of claimed cases and rates were explored using the chi-square test for trends.

We performed a time series analysis, using the Korean population from the Korea Census Statistics (http://kostat.go.kr/portal/english/index.action) to forecast the annual number of claims cases and fatalities due to hyperglycemic crisis, as well as the associated cost burden. The predicted data were estimated on the basis of claims data from 2004 to 2013. We estimated the occurrence probabilities of the numbers of claims, claim rates, and fatality rates by non-linear regression. The predicted data were calculated by multiplying the occurrence probabilities by the estimated population from the Korea Census Statistics.

\section{RESULTS}

\section{Hyperglycemic crisis hospitalizations and fatalities}

Table 1 presents the numbers of hyperglycemic crisis hospitalizations and fatalities in Korea from 2004 to 2013. During this period, the total number of annual hyperglycemic crisis claims increased from 2,674 in 2004 to 5,540 in 2013. In contrast, the hospitalization rate per 1,000 diabetes cases increased through 2006 and then decreased from 2006 to 2013. The statistical analysis revealed an increasing trend in hyperglycemic crisis hospitalizations $(P$ for trend $<0.01)$ and a decreasing trend in the hospitalization rate per 1,000 diabetes cases $(P$ for trend $<0.01)$. The total number of deaths from hyperglycemic crisis was 1,179 in 2004 , corresponding to a mortality rate per 1,000 diabetes cases of 0.7841 . This number decreased to 841 by 2013 , corresponding to a mortality rate of 0.3260 per 1,000 diabetes cases. The mortality rate per 1,000 diabetes cases showed a decreasing trend $(P$ for trend $<0.0001)$. To determine the influence of hyperglycemic crisis on mortality, we calculated the case-related fatality rate at $<60$ days. In 2004, the case-related fatality rate at $<60$ days per 1,000 diabetes cases was 0.309 . This rate decreased to 0.212 in 2013 . However, no distinct linear trend in the case-related fatality rate at $<60$ days over the last decade was observed.

Table 2 shows hyperglycemic crisis hospitalizations and fatalities by sex, age, and diabetes subtype. The total number of claims cases in the last decade was 48,578. Men accounted for $52.5 \%$ of the total claims cases, while women accounted for $47.5 \%$. The number of claims cases was higher in men than in women in the last decade. The survival and mortality rates were $80.9 \%$ and $19.1 \%$, respectively. Men also accounted for a majority of both cases of survival and fatalities. The proportion of case-related fatalities occurring at $<60$ days was $59.1 \%$. The number of claims cases increased with age, with the highest number of claims cases being in the age range of 70 to 79 years. The percentage of survival decreased with age, while the percentage of fatalities at $<60$ days increased. The highest percentage of fatalities occurred in those $>80$ years of age. Among all claims cases, $58.3 \%$ were for patients with type 2 DM, $20.4 \%$

Table 1. Hyperglycemic Crisis Hospitalizations and Fatalities in Korea 2004 to 2013

\begin{tabular}{lccccccc}
\hline Year & $\begin{array}{c}\text { No. of } \\
\text { diabetes }\end{array}$ & $\begin{array}{c}\text { Hyperglycemic crisis } \\
\text { hospitalizations }^{\mathrm{a}}\end{array}$ & $\begin{array}{c}\text { Hospitalization } \\
\text { rate per } 1,000 \\
\text { diabetes cases }\end{array}$ & $\begin{array}{c}\text { Total no. of } \\
\text { mortality }\end{array}$ & $\begin{array}{c}\text { Mortality rate per } \\
1,000 \text { diabetes }^{\text {cases }}{ }^{\mathrm{b}}\end{array}$ & $\begin{array}{c}\text { Case-related } \\
\text { fatalities at less } \\
\text { than } 60 \text { days }\end{array}$ & $\begin{array}{c}\text { Case-related fatalities at } \\
\text { less than 60 days per } \\
1,000 \text { diabetes cases }\end{array}$ \\
\hline 2004 & $1,503,689$ & $2,674(0.178)$ & 1.78 & 1,179 & 0.7841 & $464(39.4)$ & 0.309 \\
2005 & $1,790,448$ & $3,493(0.195)$ & 1.95 & 1,217 & 0.6997 & $447(36.7)$ & 0.250 \\
2006 & $1,915,458$ & $4,908(0.256)$ & 2.56 & 864 & 0.4511 & $579(67.0)$ & 0.302 \\
2007 & $2,016,723$ & $5,001(0.248)$ & 2.48 & 941 & 0.4666 & $639(67.9)$ & 0.317 \\
2008 & $2,107,125$ & $5,079(0.241)$ & 2.41 & 522 & 0.2477 & $393(75.3)$ & 0.187 \\
2009 & $2,190,600$ & $4,983(0.227)$ & 2.27 & 834 & 0.3807 & $581(69.7)$ & 0.265 \\
2010 & $2,308,345$ & $5,298(0.230)$ & 2.30 & 889 & 0.3851 & $554(62.3)$ & 0.240 \\
2011 & $2,459,952$ & $5,696(0.232)$ & 2.32 & 1,002 & 0.4073 & $651(65.0)$ & 0.265 \\
2012 & $2,571,067$ & $5,906(0.230)$ & 2.30 & 978 & 0.3804 & $619(63.3)$ & 0.241 \\
2013 & $2,579,999$ & $5,540(0.215)$ & 2.15 & 841 & 0.3260 & $547(65.0)$ & 0.212 \\
\hline
\end{tabular}

Values are expressed as number (\%).

${ }^{\text {a }} P$ for trend $<0.01$; ${ }^{b} P$ for trend $<0.0001$. 
Table 2. Hyperglycemic Crisis Hospitalization and Fatality Rates Overall and According to Sex, Age, and Diabetes Subtype in Korea, 2004 to 2013

\begin{tabular}{|c|c|c|c|c|}
\hline \multirow{2}{*}{ Variable } & \multirow{2}{*}{ Claim cases } & \multirow{2}{*}{ Survival } & \multicolumn{2}{|c|}{ Fatality } \\
\hline & & & Total & Less than 60 days \\
\hline Total hospitalizations & 48,578 & $39,311(80.9)$ & $9,267(19.1)$ & $5,474(59.1)$ \\
\hline Male & $25,480(52.5)$ & $20,281(51.6)$ & $5,199(56.1)$ & \\
\hline Female & $23,098(47.5)$ & $19,030(48.4)$ & $4,068(43.9)$ & \\
\hline \multicolumn{5}{|l|}{ Age group, yr } \\
\hline$<10$ & $587(1.2)$ & $566(96.4)$ & $21(3.6)$ & $15(2.6)$ \\
\hline $10-19$ & $1,811(3.8)$ & $1,789(98.8)$ & $22(1.2)$ & $10(0.9)$ \\
\hline $20-29$ & $2,552(5.3)$ & $2,474(96.9)$ & $78(3.1)$ & $51(2.0)$ \\
\hline $30-39$ & $3,427(7.1)$ & $3,222(94.0)$ & $205(6.0)$ & $127(3.7)$ \\
\hline $40-49$ & $5,784(11.9)$ & $5,017(86.7)$ & $767(13.3)$ & $488(8.4)$ \\
\hline $50-59$ & $7,196(14.8)$ & $5,913(82.2)$ & $1,283(17.8)$ & $856(11.9)$ \\
\hline $60-69$ & $8,629(17.8)$ & $6,740(78.1)$ & $1,889(21.9)$ & $1,125(13.0)$ \\
\hline $70-79$ & $11,982(24.7)$ & $9,019(75.3)$ & $2,963(24.7)$ & $1,607(13.4)$ \\
\hline$\geq 80$ & $6,610(13.6)$ & $4,571(69.2)$ & $2,039(30.9)$ & $1,195(18.1)$ \\
\hline \multicolumn{5}{|l|}{ Diabetes subtype (ICD-10-CM code) } \\
\hline Type 1 (E1000-E1090) & 9,901 (20.4) & $8,472(21.6)$ & $1,429(15.4)$ & $837(15.3)$ \\
\hline Type 2 (E1100-E1190) & $28,325(58.3)$ & $22,474(57.2)$ & $5,851(63.1)$ & $3,396(62.0)$ \\
\hline Malnutrition-related (E1200-E1290) & $344(0.7)$ & $249(0.6)$ & $95(1.0)$ & $65(1.2)$ \\
\hline Other diabetes (E1300-E1390) & $1,278(2.6)$ & $1,020(2.6)$ & $258(2.8)$ & $164(3.0)$ \\
\hline Diabetes type unknown (E1400-E1490) & $8,730(18.0)$ & $7,096(18.1)$ & $1,634(17.6)$ & $1,012(18.5)$ \\
\hline DM, with coma (E1X0X) & $28,061(57.8)$ & $21,873(55.6)$ & $6,188(66.8)$ & $3,479(63.6)$ \\
\hline DM, with acidosis (E1X1X) & $20,517(42.2)$ & $17,438(44.4)$ & $3,079(33.2)$ & $1,995(36.4)$ \\
\hline
\end{tabular}

Values are expressed as number (\%).

ICD-10-CM, International Classification of Diseases, Tenth Revision, Clinical Modification; DM, diabetes mellitus.

were for patients with type $1 \mathrm{DM}$, and $18.0 \%$ were for patients with diabetes type unknown. Malnutrition-related and other diabetes accounted for a small percentage of cases. Among all claims cases, $57.8 \%$ and $42.2 \%$ included the ICD-10-CM codes for DM with coma and DM with acidosis, respectively.

\section{Predicted trends in hyperglycemic crisis}

Based on the annual claims data for cases of hyperglycemic crisis from 2004 to 2013, we calculated the observed and predicted annual claim rates of hyperglycemic crisis per 100,000 population by age (Fig. 1). The annual numbers of total hyperglycemic crisis cases increased from 2004 to 2013. If this rate of increase continues, the estimated annual claim rate for hyperglycemic crisis per 100,000 population will increase by 2030 . Moreover, the predicted annual claim rate also increases with age, and is higher in patients $>80$ years than in other age groups. The annual fatality rate is predicted to gradually decrease by 2030 (Fig. 2). Nonetheless, among patients $>80$ years, the annual fatality rate is expected to remain higher than in other age groups. As the predicted number of annual claims will increase by 2030 , the total cost of hyperglycemic crisis is predicted to increase sharply (Fig. 3).

\section{DISCUSSION}

This nationwide study based on claims data investigated the trends in hyperglycemic crisis hospitalizations and in- and outof-hospital mortality in the last decade. The annual number of claims cases gradually increased, while the hospitalization rate per 1,000 diabetes cases decreased. The mortality rate per 1,000 diabetes cases also decreased. As the total number of patients with diabetes has increased, the number of hyperglycemic crisis hospitalizations has also increased. However, the hospitalization rate and mortality rate have decreased, possibly due to improvements in education and treatment.

Some previous studies have assessed trends in DKA. HowevCopyright (C) 2019 Korean Endocrine Society 


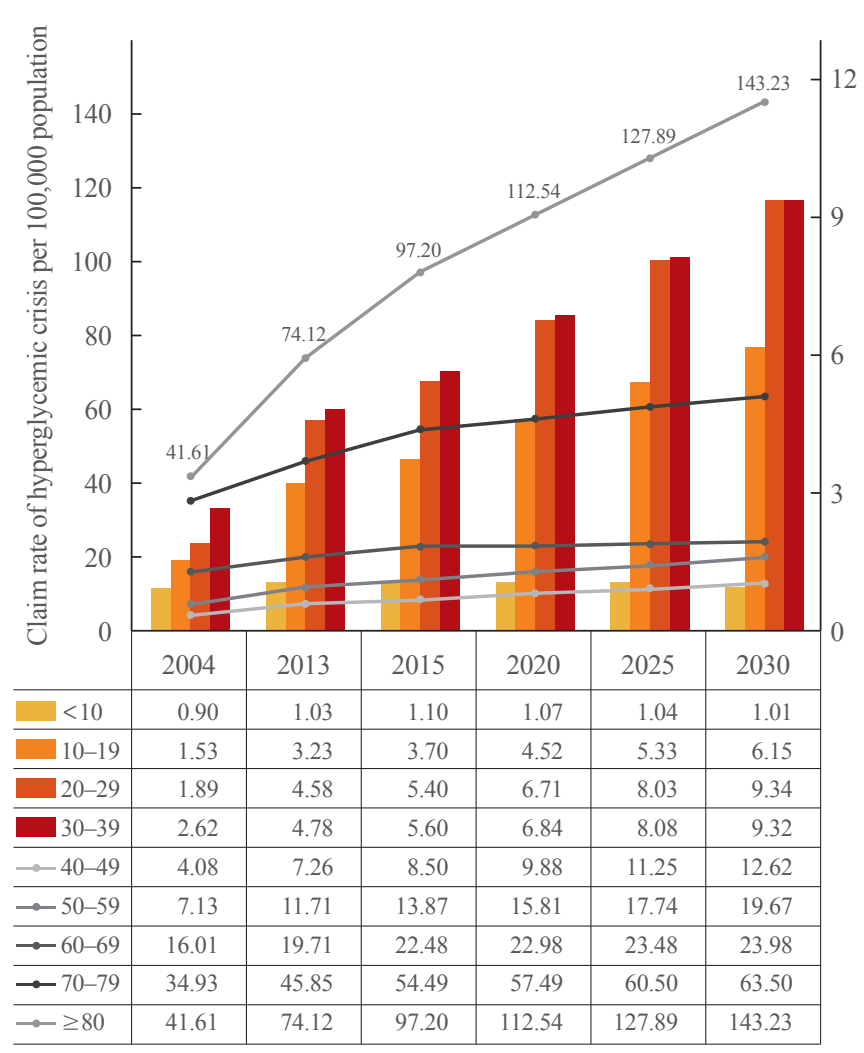

Fig. 1. Observed and predicted annual claim rate of hyperglycemic crisis per 100,000 population overall and by age.

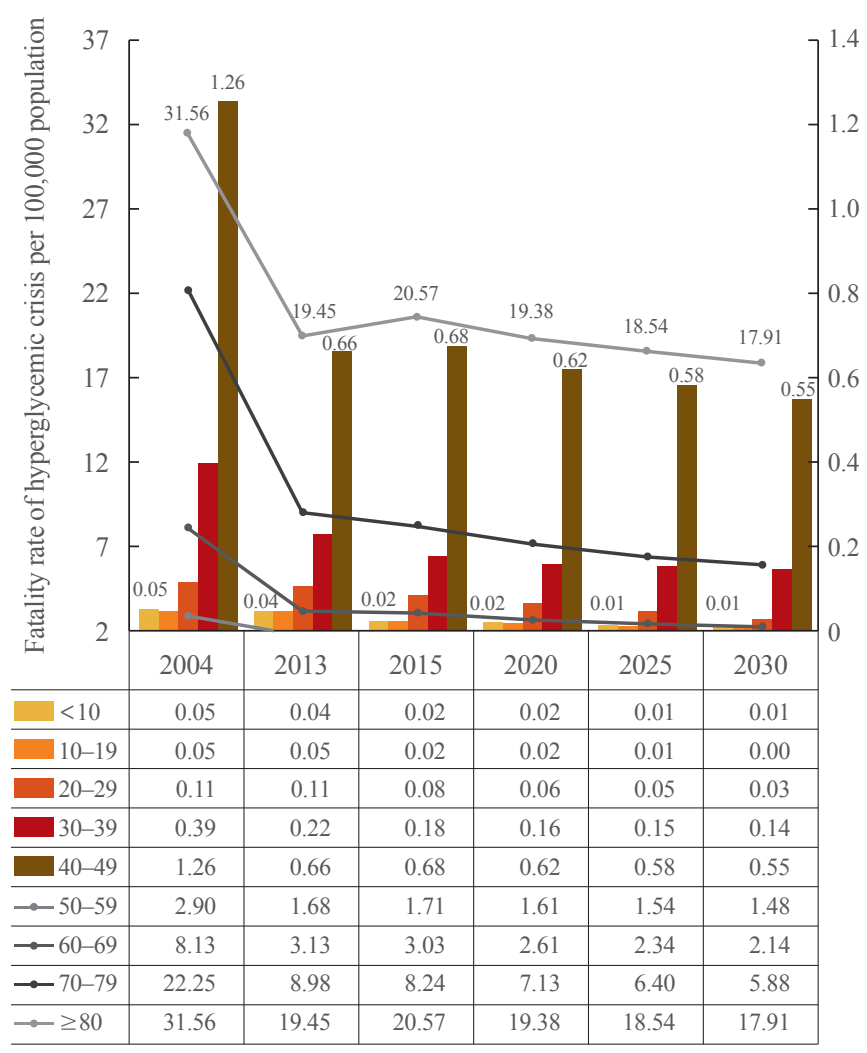

Fig. 2. Observed and predicted annual fatality rate of hyperglycemic crisis per 100,000 population overall and by age.

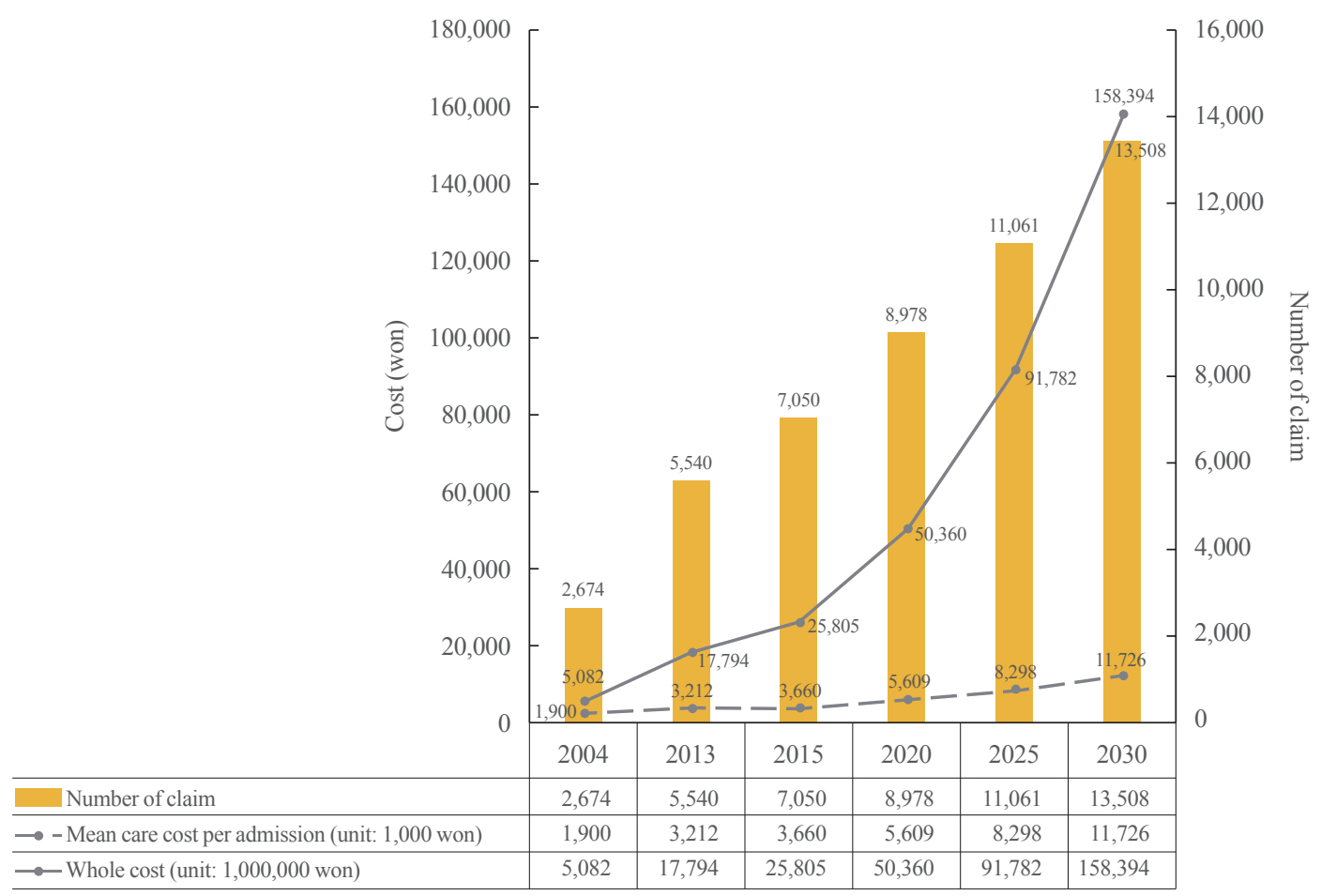

Fig. 3. Observed and predicted annual claim numbers and care costs of hyperglycemic crisis. 
er, studies of trends in hyperglycemic crisis including HHS are scarce. A recent study reported trends in the emergency department visit rate for hyperglycemic crisis in the United States [11]. The overall age-adjusted emergency department visit rate for hyperglycemic crisis was 0.7 per 100 persons in 2006 and 0.9 per 100 persons in 2011. However, there was no discernable linear trend. A retrospective medical record review in Korea showed that DKA admissions increased from 1982 to 2002 [12]. Abdulrahman et al. [13] reported that the incidence of DKA increased from 1999 to 2010 in Wales. In contrast, studies by Lombardo et al. [14] in Italy and Liu et al. [15] in Taiwan presented decreased incidence rates of DKA. The reasons for the difference between our results and those of other studies may be (1) that our study included both DKA and HHS and (2) that the quality of outpatient diabetes care varies from country to country. Regarding trends in mortality due to hyperglycemic crisis, a study in the United States revealed that deaths decreased from 1985 to 2002 [16]. Venkatesh et al. [17] and other studies [3,16] also demonstrated decreased DKA mortality rates. These results are similar to ours, and the underlying reason for this similarity is that treatments for hyperglycemic crisis have improved. The development of intensive insulin therapy, including multiple doses of insulin, has contributed to the treatment of both DKA and HHS. Studies on trends in DKA also included more subjects with type 1 DM than with type 2 DM [13,18]. DKA develops more frequently in individuals with type $1 \mathrm{DM}$ than in those with type 2 DM [8]. However, our study had more patients with type $2 \mathrm{DM}$ than with type $1 \mathrm{DM}$. Our results included all cases of HHS, and there were regional differences in DM. Previous studies have shown regional differences in the incidence of type 1 DM [19]. Therefore, our results likely differ from those of other studies in this regard because of variation across countries.

To the best of our knowledge, this is the first national population-based study in Korea to evaluate recent trends in hyperglycemic crisis. We evaluated the annual trends and quantified the increase in the annual number of claims cases. We also analyzed trends by sex and age groups. Therefore, the present study provides a more accurate and up-to-date assessment of the state of hyperglycemic crisis, which is a serious and acute complication of DM. Our study also predicted trends in hyperglycemic crisis through 2030, which will be important for making accurate estimations of the disease burden. An improved understanding of the future situation may spur investment in the disease to effectively manage the disease burden.

The current study has several limitations that should be addressed in further investigations. First, we defined subjects as having hyperglycemic crisis based on information about the diagnosis from the claims data. Only subjects who actively utilized healthcare and were able to access medical personnel were represented in the NHIS database and analyzed in this study. The claims cases for hyperglycemic crisis were not confirmed by laboratory data or medical records. Moreover, claims cases with a code of E1X0X (DM, with coma) may have included cases with coma due to hypoglycemia. Thus, there may be a gap between the actual state of hyperglycemic crisis and our results. Second, our study did not include users of sodium-glucose cotransporter-2 (SGLT2) inhibitors, which have been increasingly used in recent years for the treatment of type 2 DM. One of the adverse events of SGLT2 inhibitors is DKA [20]. Because SGLT2 inhibitors were only approved in Korea in 2014, they were not a major contributor to our results. Therefore, further research is necessary to clarify the relationship between DKA and SGLT2 inhibitor use. Lastly, our study had limitations in calculating the mortality rate of hyperglycemic crisis. Many elderly patients, who accounted for a large proportion of patients with hyperglycemic crisis, were transferred to elderly care facilities after treatment of hyperglycemic crisis. Some of the elderly died at those facilities. Therefore, it was not clear whether hyperglycemic crisis was the cause of death in older patients because we could not check individual medical records. Although we utilized mortality at $<60$ days as the cut-off point to evaluate the case-related fatality rate, this limitation of our study remains.

The number of hyperglycemic crisis hospitalizations in Korea has increased in the last decade, although the hospitalization rate per 1,000 diabetes cases and the mortality rate have decreased. As the number of patients with diabetes is increasing, the number of patients with hyperglycemic crisis, who are at a higher risk of related comorbidities, will also steadily increase. Thus, it is necessary to establish a long-term healthcare management plan for preventing hyperglycemic crisis.

\section{CONFLICTS OF INTEREST}

No potential conflict of interest relevant to this article was reported.

\section{ACKNOWLEDGMENTS}

This study was supported by a grant from the National Health Insurance Service Policy Research (2015-20-009). The funding sources were not involved in oversight or design of the study, in the analysis or interpretation of the data, or in the decision to 
submit the manuscript for publication.

\section{AUTHOR CONTRIBUTIONS}

Conception or design: S.O.S. Acquisition, analysis, or interpretation of data: S.O.S., K.H.P., J.Y.N., D.W.K., H.M.K., D.J.K., Y.L., B.W.L. Drafting the work or revising: J.H.Y., S.O.S., S. H.P. Final approval of the manuscript: J.H.Y., S.O.S.

\section{ORCID}

Ji Hong You https://orcid.org/0000-0003-1544-7094

Sun Ok Song https://orcid.org/0000-0003-4829-3407

\section{REFERENCES}

1. Huang CC, Weng SF, Tsai KT, Chen PJ, Lin HJ, Wang JJ, et al. Long-term mortality risk after hyperglycemic crisis episodes in geriatric patients with diabetes: a national population-based cohort study. Diabetes Care 2015;38:746-51.

2. Wolfsdorf J, Glaser N, Sperling MA; American Diabetes Association. Diabetic ketoacidosis in infants, children, and adolescents: a consensus statement from the American Diabetes Association. Diabetes Care 2006;29:1150-9.

3. Benoit SR, Zhang Y, Geiss LS, Gregg EW, Albright A. Trends in diabetic ketoacidosis hospitalizations and in-hospital mortality: United States, 2000-2014. MMWR Morb Mortal Wkly Rep 2018;67:362-5.

4. Malone ML, Gennis V, Goodwin JS. Characteristics of diabetic ketoacidosis in older versus younger adults. J Am Geriatr Soc 1992;40:1100-4.

5. Ramaesh A. Incidence and long-term outcomes of adult patients with diabetic ketoacidosis admitted to intensive care: a retrospective cohort study. J Intensive Care Soc 2016;17:22233.

6. Pasquel FJ, Umpierrez GE. Hyperosmolar hyperglycemic state: a historic review of the clinical presentation, diagnosis, and treatment. Diabetes Care 2014;37:3124-31.

7. Kim S. Burden of hospitalizations primarily due to uncontrolled diabetes: implications of inadequate primary health care in the United States. Diabetes Care 2007;30:1281-2.

8. Kitabchi AE, Umpierrez GE, Miles JM, Fisher JN. Hyperglycemic crises in adult patients with diabetes. Diabetes Care 2009;32:1335-43.

9. O'Shea M, Teeling M, Bennett K. The prevalence and ingredient cost of chronic comorbidity in the Irish elderly population with medication treated type 2 diabetes: a retrospective cross-sectional study using a national pharmacy claims database. BMC Health Serv Res 2013;13:23.

10. Song SO, Lee YH, Kim DW, Song YD, Nam JY, Park KH, et al. Trends in diabetes incidence in the last decade based on Korean National Health Insurance claims data. Endocrinol Metab (Seoul) 2016;31:292-9.

11. Wang J, Geiss LS, Williams DE, Gregg EW. Trends in emergency department visit rates for hypoglycemia and hyperglycemic crisis among adults with diabetes, United States, 2006-2011. PLoS One 2015;10:e134917.

12. Ko SH, Lee WY, Lee JH, Kwon HS, Lee JM, Kim SR, et al. Clinical characteristics of diabetic ketoacidosis in Korea over the past two decades. Diabet Med 2005;22:466-9.

13. Abdulrahman GO, Amphlett B, Okosieme OE. Trends in hospital admissions with diabetic ketoacidosis in Wales, 1999-2010. Diabetes Res Clin Pract 2013;100:e7-10.

14. Lombardo F, Maggini M, Gruden G, Bruno G. Temporal trend in hospitalizations for acute diabetic complications: a nationwide study, Italy, 2001-2010. PLoS One 2013;8:e63675.

15. Liu CC, Chen KR, Chen HF, Huang HL, Ko MC, Li CY. Trends in hospitalization for diabetic ketoacidosis in diabetic patients in Taiwan: analysis of national claims data, 19972005. J Formos Med Assoc 2010;109:725-34.

16. Wang J, Williams DE, Narayan KM, Geiss LS. Declining death rates from hyperglycemic crisis among adults with diabetes, U.S., 1985-2002. Diabetes Care 2006;29:2018-22.

17. Venkatesh B, Pilcher D, Prins J, Bellomo R, Morgan TJ, Bailey M. Incidence and outcome of adults with diabetic ketoacidosis admitted to ICUs in Australia and New Zealand. Crit Care 2015;19:451.

18. Henriksen OM, Roder ME, Prahl JB, Svendsen OL. Diabetic ketoacidosis in Denmark incidence and mortality estimated from public health registries. Diabetes Res Clin Pract 2007;76:51-6.

19. Song SO, Song YD, Nam JY, Park KH, Yoon JH, Son KM, et al. Epidemiology of type 1 diabetes mellitus in Korea through an investigation of the national registration project of type 1 diabetes for the reimbursement of glucometer strips with additional analyses using claims data. Diabetes Metab J 2016;40:35-45.

20. Jeon JY, Kim SK, Kim KS, Song SO, Yun JS, Kim BY, et al. Clinical characteristics of diabetic ketoacidosis in users and non-users of SGLT2 inhibitors. Diabetes Metab 2019 Jan 9 [Epub]. https://doi.org/10.1016/j.diabet.2019.01.001. 